\title{
Oscillatory decay of the survival probability of activated diffusion across a limit cycle
}

\author{
K. Dao Duc ${ }^{1,2}$ Z. Schuss $^{3}$ D. Holcman ${ }^{1,2}$ \\ ${ }^{1}$ Ecole Normale Supérieure, ${ }^{2}$ Group of Applied Mathematics and Computational Biology, IBENS, \\ 46 rue d'Ulm 75005 Paris, France ${ }^{3}$ Department of Applied Mathematics, Tel-Aviv University, Tel-Aviv, Israel.
}

(Dated: June 7, 2021)

\begin{abstract}
Activated escape of a Brownian particle from the domain of attraction of a stable focus over a limit cycle exhibits non-Kramers behavior: it is non-Poissonian. When the attractor is moved closer to the boundary oscillations can be discerned in the survival probability. We show that these oscillations are due to complex-valued higher order eigenvalues of the Fokker-Planck operator, which we compute explicitly in the limit of small noise. We also show that in this limit the period of the oscillations is the winding number of the activated stochastic process. These peak probability oscillations are not related to stochastic resonance and should be detectable in planar dynamical systems with the topology described here.
\end{abstract}

Thermal activation over a potential barrier consists in the escape of a noisy dynamical system from the domain of attraction of a stable equilibrium point of the drift, as described in Kramers' theory [1]. The activation process is the generic model of many processes in physics, chemistry, tracking, and the manifestation of many molecular and cellular processes, to mention but a few. The mean first passage time (MFPT) of the random trajectories to the boundary of the domain is a measure of the stochastic stability of the system under noisy perturbations and is determined by the depth of the potential well. In Kramers' theory the escape process is Poissonian for sufficiently long times, with rate that is one-half the reciprocal of the MFPT [2 9].

However, this is not the case for nonconservative noisy dynamics, in which the drift is not a gradient of a potential: the escape process from the domain of attraction of a meta-stable point is no longer Poissonian. We study here the noise-induced escape from the domain of attraction $D$ of a stable focus of nonconservative planar dynamics $\boldsymbol{b}(\boldsymbol{x})$ across the boundary of $D$, which is assumed to be a repelling limit cycle. This situation arises, for example, in the damped Langevin equation in the phase plane, in synchronization loops in communications theory, and more. The renewed interest in this problem is due to it manifestation in models of neuronal activity [10]. This mathematical model was considered in [7, [8] for the steady state of a system with reinjection of escaping trajectories. It was shown that for noise intensity $\varepsilon \rightarrow 0$, the arrival rate at the absorbing boundary has the asymptotic representation $R \sim \times \varepsilon^{b} G(|\log \varepsilon|) \exp \{-\Delta \psi / \varepsilon\}$, where $\Delta \psi$ is the depth of the nonequilibrium potential, the parameter $b$ is model-dependent, and the factor $G(|\log \varepsilon|)$ is a model-dependent periodic function of $|\log \varepsilon|$. It is also shown that prior to absorption in the boundary the random trajectories wind around the attractor.

We study here the time-dependent escape problem and find time-dependent oscillations of the escape rate. When the attractor is moved toward the boundary this effect is manifested through periodic peaks in the distribution of the exit time. These oscillations in the survival probabil-
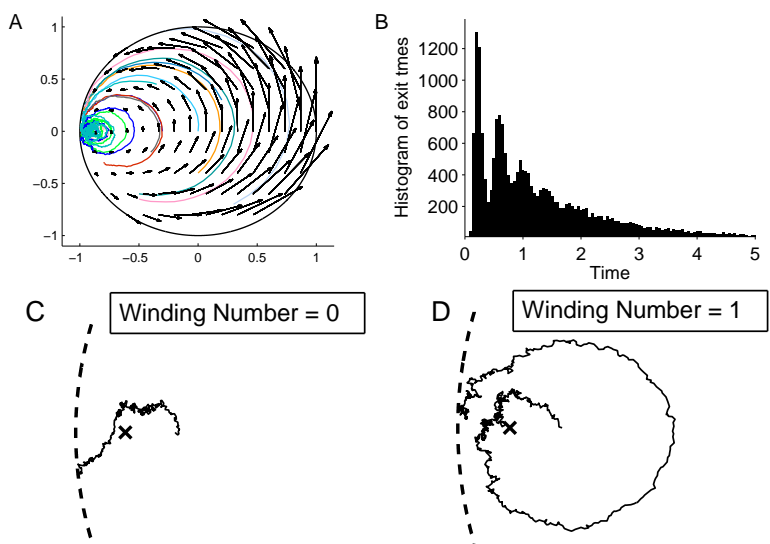

E
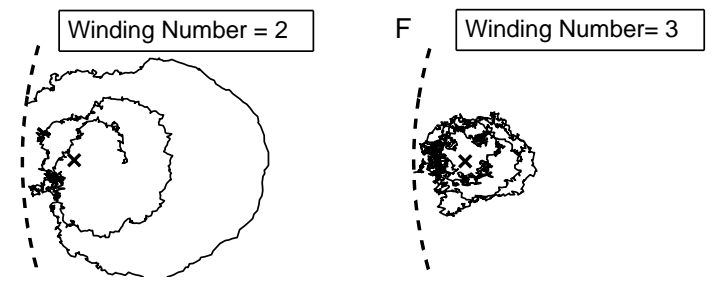

FIG. 1. Trajectories and histogram of exit times. A: Trajectories generated by (11) with the field (21) in the unit disk. B: Histogram of exit times associated to 1 from the unstable limit cycle (disk). Parameters of the simulation are $\alpha=0.9, \varepsilon=0.001, \omega=15$, number of simulations $=20000$, initial point : $(-0.5,0)$.

ity, previously mistaken for a manifestation of stochastic resonance, are shown to be the result of complex-valued higher-order eigenvalues of the Dirichlet problem for the Fokker-Planck operator inside the limit cycle. We compute the entire spectrum of the problem and show that the period of the oscillations is determined by the imaginary part of the second eigenvalue. The oscillatory peaks are observable when the real part of the second eigenvalue is comparable to the (real-valued first eigenvalue, which is the reciprocal of the MFPT. It is shown here that the oscillation of the exit probability peaks is a generic phenomenon in noise-driven planar dynamical systems that 
have the topology of the classical Hopf system. They become discernible when the focus is moved toward the repelling boundary.

Absorbing boundary and survival probability. We consider the planar stochastic dynamics

$$
\dot{\boldsymbol{x}}_{\varepsilon}(t)=\boldsymbol{b}\left(\boldsymbol{x}_{\varepsilon}(t)\right)+\sqrt{2 \varepsilon} \boldsymbol{a}\left(\boldsymbol{x}_{\varepsilon}(t)\right) \dot{\boldsymbol{w}}(t),
$$

with a drift field $\boldsymbol{b}(\boldsymbol{x})$ that has a stable focus $\boldsymbol{x}_{0}$, whose domain of attraction $D$ is bounded by an unstable limit cycle $\partial D$ (see Fig凹). Here $\dot{\boldsymbol{w}}(t)$ is $\delta$-correlated Gaussian white noise $(\boldsymbol{w}(t)$ is the Wiener process) and $\sqrt{2 \varepsilon} \boldsymbol{a}(\boldsymbol{x})$ is the diffusion matrix, scaled by a small parameter $\varepsilon$. The trajectories of (1) are terminated instantaneously the moment they hit $\partial D$ for the first time. It is well-known that even for arbitrarily small $\varepsilon>0$ the trajectories of (11) exit $D$ in finite time with probability 1 and have finite mean, called the mean first passage time $\bar{\tau}_{\varepsilon}$ (MFPT) 2[4, 6, 9 ].

The survival probability $\operatorname{Pr}_{\text {surv }}(t)$ of trajectories in $D$, prior to termination at the boundary $\partial D$, averaged with respect to an initial density distribution $p_{0}(\boldsymbol{x})$, can be expressed in terms of the transition probability density function (pdf) $p_{\varepsilon}(\boldsymbol{y}, t \mid \boldsymbol{x})$ of the surviving trajectories as

$$
\operatorname{Pr}_{\text {surv }}(t)=\int_{D} \int_{D} p_{\varepsilon}(\boldsymbol{y}, t \mid \boldsymbol{x}) p_{0}(x) d \boldsymbol{y} d \boldsymbol{x} .
$$

The pdf is the solution of the initial-boundary value problem for the Fokker-Planck equation

$$
\begin{gathered}
\frac{\partial p_{\varepsilon}(\boldsymbol{y}, t \mid \boldsymbol{x})}{\partial t}=L \boldsymbol{y} p(\boldsymbol{y}, t \mid \boldsymbol{x}) \text { for } \boldsymbol{x}, \boldsymbol{y} \in D \\
p_{\varepsilon}(\boldsymbol{y}, t \mid \boldsymbol{x})=0 \text { for } \boldsymbol{x} \in \partial D, \boldsymbol{y} \in D, t>0 \\
p_{\varepsilon}(\boldsymbol{y}, 0 \mid \boldsymbol{x})=\delta(\boldsymbol{y}-\boldsymbol{x}) \text { for } \boldsymbol{x}, \boldsymbol{y} \in D
\end{gathered}
$$

where the Fokker-Planck operator $L \boldsymbol{y}$ is given by

$$
L \boldsymbol{y}^{u}(\boldsymbol{y})=\varepsilon \sum_{i, j=1}^{2} \frac{\partial^{2}\left[\sigma^{i, j}(\boldsymbol{y}) u(\boldsymbol{y})\right]}{\partial y^{i} \partial y^{j}}-\sum_{i=1}^{2} \frac{\partial\left[b^{i}(\boldsymbol{y}) u(\boldsymbol{y})\right]}{\partial y^{i}} .
$$

Here $\boldsymbol{\sigma}(\boldsymbol{x})=\boldsymbol{a}(\boldsymbol{x}) \boldsymbol{a}^{T}(\boldsymbol{x})$. In the case at hand the operator $L \boldsymbol{y}$ with the homogeneous Dirichlet boundary conditions (3) is non-self-adjoint, has complex-valued higherorder eigenvalues $\lambda_{n, m}$, and the eigenfunctions $u_{n, m}(\boldsymbol{y})$ of $L \boldsymbol{y}$ and $v_{n, m}(\boldsymbol{x})$ of $L_{\boldsymbol{x}}^{*}$ form bi-orthonormal bases. Only the principal eigenvalue $\lambda_{0}$ is positive and so are the corresponding eigenvalues $u_{0}(\boldsymbol{y})$ and $v_{0}(\boldsymbol{x})$. The general solution of Fokker-Planck initial-boundary value problem (3) can be expanded as

$$
\begin{aligned}
p_{\varepsilon}(\boldsymbol{y}, t \mid \boldsymbol{x}) & =e^{-\lambda_{0} t} u_{0}(\boldsymbol{y}) v_{0}(\boldsymbol{x}) \\
& +\sum_{n, m} e^{-\lambda_{n, m} t} u_{n, m}(\boldsymbol{y}) \bar{v}_{n, m}(\boldsymbol{x})
\end{aligned}
$$

The probability density of the exit time $f_{\text {etd }}(t)(\mathrm{DET})$ is given by

$$
f_{\text {etd }}(t)=-\frac{d}{d t} \operatorname{Pr}_{\text {surv }}(t)=\lambda_{0} e^{-\lambda_{0} t}+\sum_{n, m} C_{n, m} e^{-\lambda_{n, m} t}(, 5)
$$

where $C_{m, n}$ are constants. It is the purpose of this letter to show that this density decays with large oscillations and we present a generic case in which they are easily discernible.

The spectrum of the non-self-adjoint FokkerPlanck operator

The field $\boldsymbol{b}(\boldsymbol{x})$. The local geometry of the drift field $\boldsymbol{b}(\boldsymbol{x})$ near the focus $\boldsymbol{x}_{0}$ and the unstable limit cycle $\partial D$ can be described as follows. Near $\boldsymbol{x}_{0}$ the local behavior of $\boldsymbol{b}(\boldsymbol{x})$ is $\boldsymbol{b}(\boldsymbol{x})=\boldsymbol{B}\left(\boldsymbol{x}-\boldsymbol{x}_{0}\right)+O\left(\left|\boldsymbol{x}-\boldsymbol{x}_{0}\right|^{2}\right)$ where the matrix $\boldsymbol{B}$ has eigenvalues in the left half of the complex plane. The local representation of the field $\boldsymbol{b}(\boldsymbol{x})$ in the boundary strip is given by

$$
\boldsymbol{b}_{\alpha}(\rho, s)=-\rho b^{0}(s) \boldsymbol{n}+B(s) \boldsymbol{t}
$$

the tangential component of the field at $\partial D$ is $B(s)=$ $\boldsymbol{b}(0, s) \cdot \nabla s=|\boldsymbol{b}(\boldsymbol{x}(s))|>0$ and the normal derivative of the normal component is $b^{0}(s) \geq 0$ for all $0 \leq s \leq 2 \pi$.

WKB structure of the pdf for small $\varepsilon$. To compute the spectrum of $L \boldsymbol{y}$, we use matched asymptotics 11] to construct a uniform asymptotic approximation to the eigenfunctions $u_{m, n}(\boldsymbol{y})$. We begin with the outer expansion of and eigenfunction $u(\boldsymbol{y})$ in the WKB form

$$
u(\boldsymbol{y})=K_{\varepsilon}(\boldsymbol{y}) \exp \left\{-\frac{\psi(\boldsymbol{y})}{\varepsilon}\right\},
$$

where the eikonal function $\psi(\boldsymbol{y})$ is a solution of eikonal equation

$$
\boldsymbol{\sigma}(\boldsymbol{y}) \nabla \psi(\boldsymbol{y}) \cdot \nabla \psi(\boldsymbol{y})+\boldsymbol{a}(\boldsymbol{y}) \cdot \nabla \psi(\boldsymbol{y})=0
$$

(see [9, Chap.10]) and $\psi(\boldsymbol{y})$ is constant on the boundary. The function $K_{\varepsilon}(\boldsymbol{y})$ is a regular function of $\varepsilon$ for $\boldsymbol{y} \in D$, but has to develop a boundary layer to satisfy the homogenous Dirichlet boundary condition $K_{\varepsilon}(\boldsymbol{y})=$ 0 for $\boldsymbol{y} \in \partial D$. Therefore $K_{\varepsilon}(\boldsymbol{y})$ is further decomposed into the product

$$
K_{\varepsilon}(\boldsymbol{y})=\left[K_{0}(\boldsymbol{y})+\varepsilon K_{1}(\boldsymbol{y})+\cdots\right] q_{\varepsilon}(\boldsymbol{y}),
$$

where $K_{0}(\boldsymbol{y}), K_{1}(\boldsymbol{y}), \ldots$ are regular functions in $D$ and on its boundary and are independent of $\varepsilon$, and $q_{\varepsilon}(\boldsymbol{y})$ is a boundary layer function. The boundary layer function $q_{\varepsilon}(\boldsymbol{y})$ satisfies the boundary condition $q_{\varepsilon}(\boldsymbol{y})=0$ for $\boldsymbol{y} \in$ $\partial D$, the matching condition $\lim _{\varepsilon \rightarrow 0} q_{\varepsilon}(\boldsymbol{y})=1$ for all $\boldsymbol{y} \in$ $D$.

To find the boundary layer equation, we introduce the stretched variable $\zeta=\rho / \sqrt{\varepsilon}$ and define $q_{\varepsilon}(\boldsymbol{x})=$ $Q(\zeta, s, \varepsilon)$. Expanding all functions in (7) in powers of $\varepsilon$ we find

$$
Q(\zeta, s, \varepsilon) \sim Q^{0}(\zeta, s)+\sqrt{\varepsilon} Q^{1}(\zeta, s)+\cdots,
$$


and we obtain the boundary layer equation

$$
\begin{aligned}
\sigma(s) \frac{\partial^{2} Q^{0}(\zeta, s)}{\partial \zeta^{2}} & -\zeta\left[b^{0}(s)+2 \sigma(s) \phi(s)\right] \frac{\partial Q^{0}(\zeta, s)}{\partial \zeta} \\
& -B(s) \frac{\partial Q^{0}(\zeta, s)}{\partial s}=0
\end{aligned}
$$

where $\sigma(s)=(\sigma(0, s) \boldsymbol{n} . \boldsymbol{n})$. The solution that satisfies the boundary and matching conditions $Q^{0}(0, s)=$ $0, \quad \lim _{\zeta \rightarrow-\infty} Q^{0}(\zeta, s)=1$ is given by

$$
Q^{0}(\zeta, s)=-\sqrt{\frac{2}{\pi}} \int_{0}^{\xi(s) \zeta} e^{-z^{2} / 2} d z
$$

where $\xi(s)$ is the $S$-periodic solution of the Bernoulli equation

$$
\sigma(s) \xi^{3}(s)+\left[b^{0}(s)+2 \sigma(s) \phi(s)\right] \xi(s)+B(s) \xi^{\prime}(s)=0 .
$$

The principal eigenvalue. The principal eigenvalue is the reciprocal of the MFPT $\bar{\tau}_{\varepsilon}$ of $\boldsymbol{x}_{\varepsilon}(t)$ to $\partial D$, that is, $\lambda_{0} \sim 1 / \bar{\tau}_{\varepsilon}$. When $\lambda_{0}$ is exponentially small, it determines the slow decay of the survival probability. For small $\varepsilon$ the $\mathrm{MFPT} \bar{\tau}_{\varepsilon}$ is given by

$$
\bar{\tau}_{\varepsilon}=\frac{\pi^{3 / 2} \sqrt{2 \varepsilon}}{\sqrt{\operatorname{det} \boldsymbol{H}} \int_{0}^{2 \pi} K_{0}(0, s) \xi(s) d s} \exp \left\{\frac{\hat{\psi}}{\varepsilon}\right\}
$$

where $\boldsymbol{H}$ the solution of the Riccati equation $2 \boldsymbol{H} \boldsymbol{\sigma}\left(\boldsymbol{x}_{0}\right) \boldsymbol{H}+\boldsymbol{H} \boldsymbol{B}+\boldsymbol{B}^{T} \boldsymbol{H}=\mathbf{0}$, the function $\xi(s)$ is defined in (13), and [6]

$$
K_{0}(0, s)=\frac{\xi(s)}{B(s)} .
$$

The full spectrum. To compute the higher order eigen$\overline{\text { functions, we set } \eta}=\xi(s) \zeta$ and obtain the boundary layer equations

$$
\begin{aligned}
\frac{\partial^{2} \tilde{Q}^{0}(\eta, s)}{\partial \eta^{2}} & +\eta \frac{\partial \tilde{Q}^{0}(\eta, s)}{\partial \eta}+\frac{B(s)}{\sigma(s) \xi^{2}(s)} \frac{\partial \tilde{Q}^{0}(\eta, s)}{\partial s} \\
& =-\frac{\lambda}{\sigma(s) \xi^{2}(s)} \tilde{Q}^{0}(\eta, s) .
\end{aligned}
$$

Separating $\tilde{Q}^{0}(\eta, s)=R(\eta) T(s)$, we obtain for the even function $R(\eta)$ the eigenvalue problem

$$
R^{\prime \prime}(\eta)+\eta R^{\prime}(\eta)+\mu R(\eta)=0, R(0)=0, \lim _{\eta \rightarrow-\infty} R(\eta)=0,
$$

where $\mu$ is the separation constant and the eigenvalues are $\mu_{n}=2 n,(n=1,2, \ldots)$ with the eigenfunctions $R_{n}(\eta)=\exp \left\{-\frac{\eta^{2}}{2}\right\} H_{2 n+1}\left(\frac{\eta}{\sqrt{2}}\right)$, where $H_{2 n+1}(x)$ are the Hermite polynomials of odd orders [12]. The function $T(s)$ is given by

$$
T(s)=\exp \left\{-\lambda \int_{0}^{s} \frac{d s^{\prime}}{B\left(s^{\prime}\right)}+2 n \int_{0}^{s} \frac{\sigma\left(s^{\prime}\right) \xi^{2}\left(s^{\prime}\right)}{B\left(s^{\prime}\right)} d s^{\prime}\right\} .
$$

The $2 \pi$-period gives for $n=1, \ldots$, the eigenvalues

$$
\begin{aligned}
\lambda_{m, n} & =\left[\frac{n}{\pi} \int_{0}^{2 \pi} \frac{\sigma(s) \xi^{2}(s)}{B(s)} d s+m i\right] \tilde{\omega} . \\
\tilde{\omega} & =\frac{2 \pi}{\int_{0}^{2 \pi} \frac{d s}{B(s)}} .
\end{aligned}
$$

Thus the expressions (14), and (18) define the full spectrum as

$$
S p(L)=\left\{\lambda_{0}[1+O(\varepsilon)], \bigcup_{\substack{n \geq 0 \\ m= \pm 1, \pm 2, \ldots}} \lambda_{m, n}[1+O(\varepsilon)]\right\} .
$$

A generic model. To illustrate the theory, we compute the exit distribution of a generic stochastic system when the focus $\boldsymbol{x}_{0}$ is moved close to the limit cycle $\partial D$. Oscillations in the probability density of the exit time (5) become discernible (see Fig.1B), as shown in the following example: the complex plane Hopf-system

$$
\boldsymbol{b}(z)=z\left(-1+|z|^{2}+i \omega\right)
$$

where $\omega>0$ is the angular velocity of the field, has a focus at the origin and its unstable limit cycle is the unit circle. The Möbius transformation

$$
\Phi_{\alpha}(z)=\frac{z-\alpha}{1-\alpha z}, \quad 0<\alpha<1,
$$

moves the focus to $\boldsymbol{x}_{0}=(-\alpha, 0)$ and leaves the limit cycle invariant. The resulting field is given by

$$
\boldsymbol{b}_{\alpha}(z)=\frac{(z+\alpha)(1+\alpha z)}{\left(1-\alpha^{2}\right)}\left(-1+\left|\frac{z+\alpha}{1+\alpha z}\right|^{2}+i \omega\right)
$$

The decomposition (6) is given by

$$
\boldsymbol{b}_{\alpha}(\rho, \theta)=-\rho\left[b_{\alpha}^{0}(\theta)+O\left(\rho^{2}\right)\right] \boldsymbol{\nu}+b_{\alpha}^{*}(\rho, \theta) \boldsymbol{t},
$$

where $\boldsymbol{t}$ and $\boldsymbol{\nu}$ are the unit tangent and outer-normal to $\partial D$ at $(0, \theta)$, respectively. The components $b_{\alpha}^{0}(\theta)$ and $b_{\alpha}^{*}(\rho, \theta)$ are given by

$$
\begin{aligned}
b_{\alpha}^{0}(\theta) & =\frac{2\left(1-\alpha^{2}-\omega \alpha \sin (\theta)\right)}{1-\alpha^{2}}+O(\rho), \\
b_{\alpha}^{*}(0, \theta) & =\frac{\omega}{1-\alpha^{2}}\left(1+2 \alpha \cos (\theta)+\alpha^{2}\right)+O(\rho) .
\end{aligned}
$$

The long-time probability density $P_{\alpha}(\theta)$ of exit points on $\partial D$ is determined by the principal eigenfunction (12) and the $2 \pi$-periodic solution $\xi_{\alpha}(s)$ of the Bernoulli equation (13). In the case at hand (13) takes the form

$$
-\sigma(\theta) \xi_{\alpha}^{3}(\theta)+\left[b_{\alpha}^{0}(\theta)\right] \xi_{\alpha}(\theta)+B_{\alpha}(\theta) \xi_{\alpha}^{\prime}(\theta)=0
$$

and $\sigma(\theta)=\boldsymbol{\sigma}(0, \theta) \boldsymbol{\nu}(0, \theta) \cdot \boldsymbol{\nu}(0, \theta)$. The pdf $P_{\alpha}(\theta)$ is given by [6], [9, eq.(10.127)]

$$
P_{\alpha}(\theta)=\frac{\frac{\xi_{\alpha}^{2}(\theta) \sigma(\theta)}{b_{\alpha}^{*}(0, \theta)}}{\int_{0}^{2 \pi} \frac{\xi_{\alpha}^{2}(s) \sigma(s)}{b_{\alpha}^{*}(0, s)} d s}
$$


which leads after computations to

$$
P_{\alpha}(\theta)=\frac{\left(1+2 \alpha \cos \theta+\alpha^{2}\right)^{-3}}{\int_{-\pi}^{\pi}\left(1+2 \alpha \cos s+\alpha^{2}\right)^{-3} d s} .
$$

As $\alpha \rightarrow 1$, the density $P_{\alpha}(\theta)$ concentrates at $\theta=\pi$ (Fig 2). Brownian simulations show that the distribution of exit points on $\partial D$ is almost zero, except for a small interval $R(\alpha)$ centered at the boundary point closest to focus. This result shows that exit occurs only in a
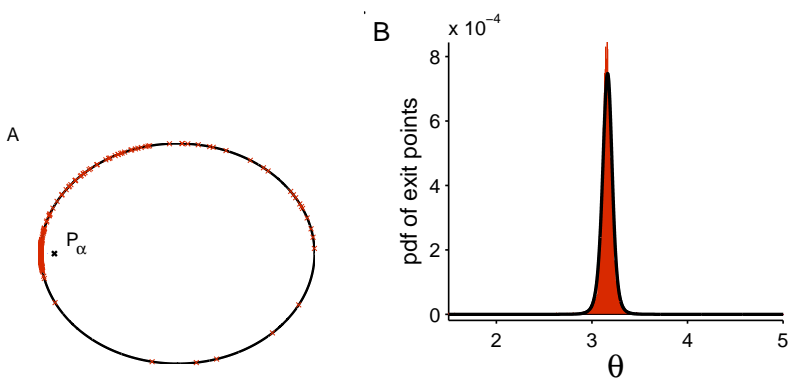

FIG. 2. Density of exit points.(Left): Exit points are marked red. (Right) Histogram of exit points (red) and the density $P_{\alpha}(\theta)$ from (24) (black). Simulation parameters are $\alpha=-0.9, \omega=10, \varepsilon=0.005$. Number of runs $=50000$.

small arc of the boundary. When a trajectory fails to hit the boundary while in the neighborhood of this arc, it has to wind around the focus and return to $R(\alpha)$. In the Brownian dynamics simulations of the noisy Hopf system we followed the history of each trajectory and evaluated its contribution to the exit time distribution by recording its winding number prior to exit. The winding renders the average lengths of exiting trajectories quantized by the winding numbers around the focus, as confirmed in the empirical statistics presented in Fig 3

To evaluate the MFPT (14), we find that $\boldsymbol{H}_{\alpha}=\boldsymbol{I}$, $\hat{\psi}_{\alpha}=\psi_{\alpha}(-1)=\frac{1}{2}(1-\alpha)^{2}$, and direct integration gives

$$
\begin{aligned}
& \int_{0}^{2 \pi} K_{0}(0, s) \xi_{\alpha}(s) d s=\frac{4 \pi\left(\alpha^{4}+4 \alpha^{2}+1\right)}{C(\omega)\left(1+\alpha^{2}\right)}, \\
& C(\omega)=\frac{3 \omega}{8}-\frac{8 / \omega}{1+(4 / \omega)^{2}}+\frac{4 / \omega}{\left.4+(4 / \omega)^{2}\right)}>0 .
\end{aligned}
$$

It follows that for $(1-\alpha)^{2} / 2 \varepsilon=O(1)$,

$$
\begin{aligned}
\bar{\tau}_{\varepsilon} & \sim \frac{C(\omega) \sqrt{2 \pi \varepsilon}(1+\alpha)^{2}}{4\left(1+4 \alpha^{2}+\alpha^{4}\right)} \exp \left\{\frac{\hat{\psi}_{\alpha}}{\varepsilon}\right\} \\
& \sim \frac{C(\omega) \sqrt{2 \pi \varepsilon}}{6} \exp \left\{\frac{(1-\alpha)^{2}}{2 \varepsilon}\right\}=O(1),
\end{aligned}
$$

so it is not exponentially long in $\varepsilon^{-1}$ as in Kramers' and the classical exit problems.

The second eigenvalue. To determine the second eigenvalue from (18), we note that here $b_{\alpha}^{*}(0, \theta)=\omega, b_{0}^{0}(\theta)=2$,
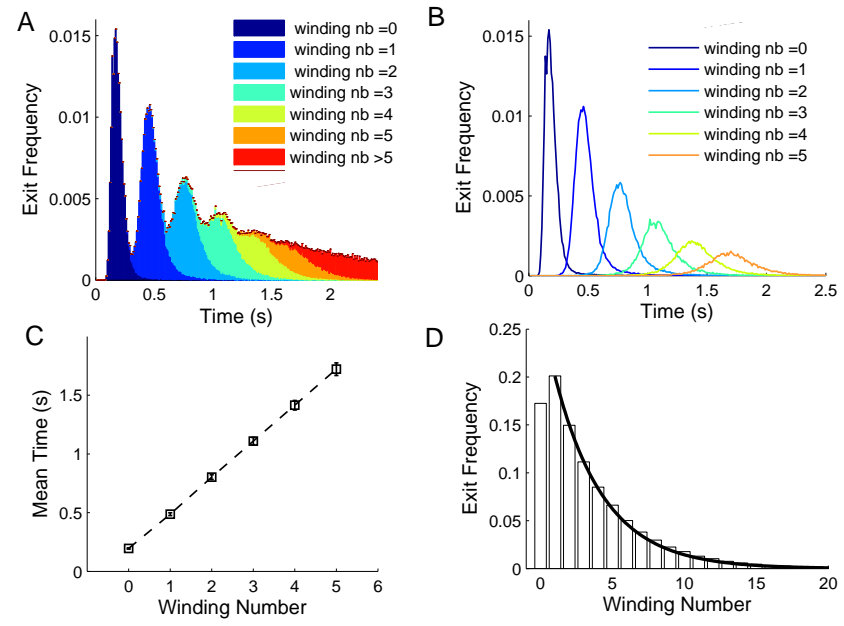

FIG. 3. Statistic of exit trajectories and winding. A: The different colors represent exit frequencies with different winding numbers. B: Exit frequencies conditioned on winding number (0 to 5 turns). C: Mean of exit times in histogram $B$ vs winding number. D: Histogram of winding numbers in A. The exponential function $f(n)=p(1-p)^{n-1}$ (solid line) approximates the decay rate. Here $p$, the ratio of frequencies of 1 to 2 turns, is the probability to exit without making a turn.

so $\tilde{\omega}=\omega$ and $\omega_{1}(\alpha)=4$. We conclude with the surprising result that the period of the peak in $f_{\text {etd }}(t)$ is $2 \pi / \omega$, where $\omega$ is precisely the frequency at the focus point. Furthermore, using Brownian simulations, we found that $f_{\text {etd }}(t)$ can be well approximated by the sum of the first two exponentials $\tilde{f}_{\text {etd }}(t)=C_{0} e^{-\lambda_{0} t}+C_{1} e^{-\omega_{1} t} \cos (\omega t+\phi)$, where $\lambda_{0}$ is the principal eigenvalue and $C_{0}, C_{1}, \phi$ are constants.

\section{Discussion and conclusion}

We have demonstrated that the oscillation of the exit distribution is an intrinsic property of dynamical systems driven by noise. As the focus is moved toward the limit cycle, the oscillations become discernible. The frequency of the peaks is the oscillation frequency of the deterministic dynamical system near the attractor. This is surprising, because the oscillation is not affected by the noise amplitude. As the focus moves toward the limit cycle, the first eigenvalue becomes of order 1 , but also the second eigenvalue is of order one and does not change. This phenomenon is observable in a class of escape problems from the domain of attraction of a stable focus across the unstable limit cycle bounding the domain. Richer phenomenology should be expected in dimensions higher than two. Note that the peak oscillations are not related to stochastic resonance and can potentially be used to interpret physical and biological escape phenomena from their physical model. 
[1] Kramers H.A., Physica, 7, pp.284-304 (1940).

[2] Matkowsky, B.J. and Z. Schuss, SIAM J. of Appl. Math. 33, pp.365-382 (1977).

[3] Freidlin, M.I., Wentzell, A.D. Random Perturbations of Dynamical Systems. Grundlehren der Mathematischen Wissenschaften 260 (Second edition). Springer-Verlag, NY 1998.

[4] Hänggi, P., P. Talkner, M. Borkovec, Rev. Mod. Phys 62, pp.251-341 (1990).

[5] Gang H. et al. Phys. Rev. Lett. 71, 807 - 810 (1993)

[6] Matkowsky, B.J., Z. Schuss, SIAM J. of Appl. Math. 42 (4), pp.822-834 (1982).
[7] Maier, R.S. and D.L. Stein, Phys. Rev. Lett. 77 (24), pp.4860-4863 (1996).

[8] Maier, R.S. and D.L. Stein, SIAM J. Appl. Math. 57 (3), pp.752-790 (1997).

[9] Schuss, Z. Diffusion and Stochastic Processes: an Analytical Approach. Springer series on Applied Mathematical Sciences vol.170, Springer NY 2010.

[10] Holcman, D. and M. Tsodyks, PLOS Comp. Biology 2 (3): e23 (2006).

[11] Bender, C.M. and S.A. Orszag. Advanced Mathematical Methods for Scientists and Engineers. McGraw-Hill, New York, 1978.

[12] Abramowitz, M. and I. Stegun, Handbook of Mathematical Functions with Formulas, Graphs, and Mathematical Tables, Dover Publications, NY 1972. 